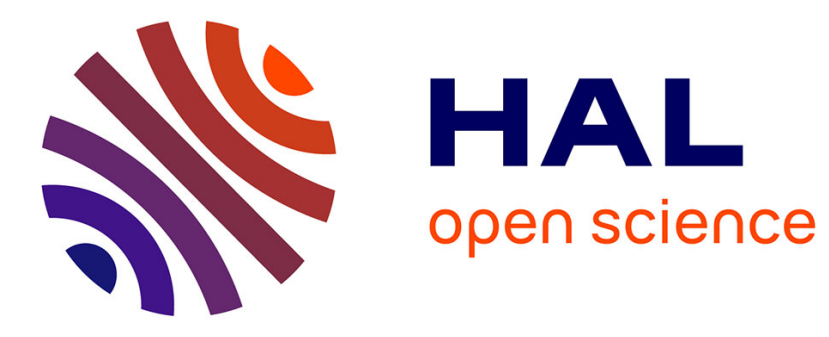

\title{
Issues of Culture in Designing for Accessibility
}

Helen Petrie, Gerhard Weber, Charudatta Jadhav, Jennifer S. Darzentas

\section{To cite this version:}

Helen Petrie, Gerhard Weber, Charudatta Jadhav, Jennifer S. Darzentas. Issues of Culture in Designing for Accessibility. 16th IFIP Conference on Human-Computer Interaction (INTERACT), Sep 2017, Bombay, India. pp.55-67, 10.1007/978-3-319-92081-8_6 . hal-01821414

\section{HAL Id: hal-01821414 \\ https://hal.inria.fr/hal-01821414}

Submitted on 22 Jun 2018

HAL is a multi-disciplinary open access archive for the deposit and dissemination of scientific research documents, whether they are published or not. The documents may come from teaching and research institutions in France or abroad, or from public or private research centers.
L'archive ouverte pluridisciplinaire HAL, est destinée au dépôt et à la diffusion de documents scientifiques de niveau recherche, publiés ou non, émanant des établissements d'enseignement et de recherche français ou étrangers, des laboratoires publics ou privés.

\section{(c)(1)}

Distributed under a Creative Commons Attribution| 4.0 International License 


\title{
Issues of Culture in Designing for Accessibility
}

\author{
Helen Petrie ${ }^{1}$, Gerhard Weber ${ }^{2}$, Charudatta Jadhav ${ }^{3}$ and Jennifer S. Darzentas ${ }^{1}$ \\ ${ }^{1}$ Department of Computer Science, University of York, United Kingdom \\ ${ }^{2}$ Department of Computer Science, Technische Universität Dresden, Germany \\ ${ }^{3}$ Accessibility and Inclusive Design Group, Tata Consultancy Services, India \\ helen.petrie@york.ac.uk, gerhard.weber@tu-dresden.de, \\ charudatta.jadhav@tcs.com, jenny.darzentas@york.ac.uk
}

\begin{abstract}
Cross cultural differences and cultural sensitivities have not yet received much attention in the areas of accessibility, assistive technologies, and inclusive design and methods for working with disabled and older users. However it is important to consider the challenges of developing accessible and usable technologies for people with disabilities and older people in different cultural contexts. This chapter presents the background to the topic and then considers three particular issues in relation to the topic: the accessibility of interactive systems in the home and implications for emerging markets; the accessibility problems in relation to a multilingual society such as India; and finally, the issues of the cultural biases of the methods used when working with users within a user centered design lifecycle or a "double diamond" methodology, whether they are mainstream users, disabled or older users.
\end{abstract}

Keywords: Cross Cultural Differences, Cultural Sensitivities, Accessibility, Universal Design, Assistive Technology, Users with Disabilities, Older Users

\section{Introduction}

The population of older people and people with disabilities is growing rapidly throughout the world, due to many complex changes in societies from decreasing birth rates to increasing survival rates from accidents and chronic health conditions. According to the World Health Organization (WHO), there are currently between 110 and 190 million people with substantial disabilities [1] and approximately 901 million older adults (usually taken to be people aged 60 or older, although the definition itself varies from country to country and organization to organization), worldwide. The United Nations (UN) predicts that the population of older adults will increase to more than 2 billion by 2050 [2]. The UN also predicts that the number of older people will exceed the number of the young people, aged 15 or younger, for the first time in 2047. This change in the balance of older to younger people (known as the old age dependency ratio or the potential support ratio) has many consequences, one of them being that older adults and people with disabilities will need to live more independently, without as many people of working age to care for them. Many analysts and researchers [e.g. 3] believe that technology will provide at least a partial solution to this problem, 
allowing older adults to live in their own homes safely and independently for as long as possible and to give disabled people greater independence. While this may be a viable solution for some individuals in the wealthy, developed countries, for older and disabled people in many parts of the world, there are issues of affordability as well as accessibility and acceptability.

These demographic changes are worldwide phenomena, although different countries are experiencing them at different rates and in different ways. In addition, there are many cultural sensitivities and differences in attitudes to disability and old age, which have important implications for designing interactive systems for disabled and older people. However, designing for cultural diversity is an aspect of universal design and can increase the overall number of users and the usability of interactive systems for those users [4].

In this chapter we explore three particular issues in relation to the topic of cultural differences and accessibility: the accessibility of interactive systems in the home and implications for emerging markets; the accessibility problems in relation to a multilingual society such as India; and finally, the issues of the cultural biases of the methods used when working with users within a user centred design lifecycle or a "double diamond" methodology, whether they are mainstream users, disabled or older users.

\section{Topic 1: Cultural differences and accessibility of interactive systems in the home}

Major drivers for the greater use of technology in the home include an interest in greater comfort and entertainment, a desire to reduce and simplify effortful and repetitious tasks, a desire to increase personal safety, interest in monitoring one's physiological state, and a desire to improve communication with family and friends. While this comes at the price of more complexity of the functionality of household technologies, there is now an abundance of products to support these functions. Many of them use a computer and serve multiple purposes through an interactive user interface. Understanding the needs of people using such interactive systems is crucial to detect both cultural and accessibility requirements in everyday activities.

Mismatches between the language and cultural assumptions of designers and those of users may lead to inappropriate functionality and hence a lack of acceptance of technologies. As pointed out by Chavan et al [5], a very small error in the design of a washing machine for the emerging markets such as India, combined with a poor understanding of Indian clothing resulted in many items of clothing being destroyed and the loss of an important market for Whirlpool in India (this in spite of excellent washing system, serviced by men, which is available in India, see Fig. 1, below). Whirlpool renamed the "delicate cycle" on their washing machine as "sari cycle" which probably seemed very culturally appropriate at the time. But unfortunately, the washing machine actually shredded the delicate fabrics of saris - Whirlpool had failed to research the cultural and linguistic conventions of this particular market.

Lack of accessibility relates to many facets of the use of computers by people with sensory, physical of cognitive needs in both developed and developing countries. In emerging countries such as China, some $73 \%$ of households already had a washing 
machine by 2010 [6]. To prevent accidental water flooding, it is mandatory to push a button to release an electromagnetic door lock before the washing machine door will open. Washing machines enter such a state at the end of the washing program and usually signal this only visually. Lack of sight makes it hard to know why the door will not open when this visual indicator is not present. Some machines do include an auditory signal, but there is only one auditory signal (a generic beep), which sounds when the washing program begins, when there is a problem and when the program ends. Although a visually impaired user can hear the signal, it is not sufficiently communicative to understand what state the washing machine is in.

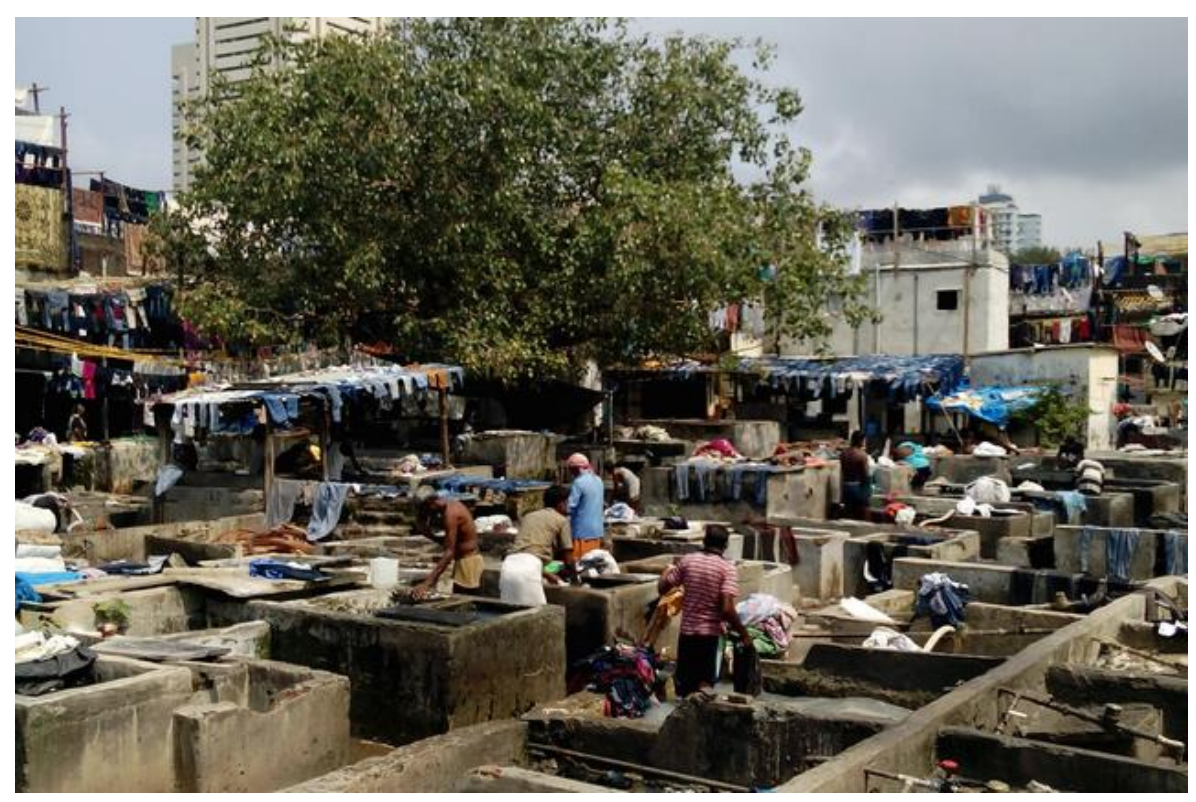

Fig. 1. Washing men at work in Mumbai (Photo $\subset$ Gerhard Weber)

At least three quarters of the world population now have mobile or smart phones [7]. In 2016, more than $98 \%$ had phones with either Android or iOS as the operating system [8]. Controlling a robot vacuum cleaner, such as the Roomba [9] by an app on a smart phone is convenient, and more importantly, the only way to change the robot's operation. However, although a robot vacuum cleaner is very convenient for people with physical disabilities such as a tremor in their hands, adjusting its setting via the app can be very difficult. Designers of such apps need to understand the requirements for larger buttons and split the virtual keyboard to reflect sub-tasks on smaller screens, if more space is needed. The same approach allows enlargement of fonts for increased readability for people who are dyslexic.

In China, Brazil and India demand for web-connected smart TVs is considerably higher than it is in developed countries [10]. However, there are a number of accessibility issues in interacting with a smart TV. For example, smart TVs encourage interaction through gesture and voice commands. For people with physical disabilities 
interacting through gesture may not be possible. Voice commands may be a useful alternative, but creating voice command interaction that avoids accidental activation of commands when simply speaking is still not easy to design. In addition, voice command interaction has not currently addressed the needs of people with speech difficulties.

The examples discussed above make it clear that specific home technologies increase quality of life in many ways but may introduce new barriers, both cultural and in relation to accessibility. Providing a range of new modalities for interacting provide good solutions for the accessibility issues. Developing countries may sometimes utilize technology considered already out-dated in the developed world, but more often are now jumping straight to the very latest technologies and skipping what might be considered the intermediate steps. For example, in Cambodia smartphone use is very high, with over $95 \%$ of citizens having access to a mobile phone of some type [11]. This is largely because the landline infrastructure was badly damaged and became very outdated during the Khmer Rouge regime and subsequent war with Vietnam (1975 1992). At the end of that period, rather than invest in landline infrastructure, investment was made in mobile infrastructure. One consequence of this is that internet access is largely via smartphones, with $80 \%$ of those Cambodians using the internet doing so via a smartphone in 2017. This is far highly than in the United Kingdom, where in 2016, $66 \%$ of citizens reported accessing the internet from their smartphone [12]. Thus, Cambodia can be seen as more technologically advanced than the UK in terms of devices for internet access.

To some extent, addressing accessibility issues can also benefit cross cultural issues. For example, designers of medical devices have understood the need for high accessibility of their devices. Speaking thermometers, large print glucose meters, talking blood measure monitors are available at low to modest costs due to their widespread use. However, embedding them in homes alongside accessible home appliances requires a range of interdisciplinary skills involving architects, hardware builders, software and industrial designers. There is a need to establish appropriate services for living independently as none of these devices have a common approach to accessibility. Making the accessibility of devices compatible can be achieved most easily through personalization capabilities [13], which may be most beneficial to address the various cultural needs of people in emerging countries.

People with disabilities and older people have needs and wishes for technology that HCI designers and developers are often unaware of or fail to understand fully. In European countries, we have observed that designers and developers of interactive systems also often have difficulty establishing contact with disabled and older people and lack a good range of techniques to work with them in the development process, in spite of being eager to develop technologies to support these user groups. There are also many cultural and societal differences between countries which affect needs and attitudes towards technologies for disabled and older people. For example, the effects of demographic changes in China are comparable to European countries, but attitudes to family are typically somewhat different from European attitudes. Even between European and North American countries that are considerable differences in attitudes to disabled and older people. In the Nordic countries, there is a very strong philosophy that the whole of society has a responsibility to provide quality of life and opportunities for disabled people and to care for older people. In southern European countries, the philosophy is more that the responsibility lies with the individual family to care for 
their family members. In North America disabled people are strongly encouraged to be very independent whereas in European countries the philosophy if more of providing as much support as possible (e.g. financially, technically) for disabled people.

When designing an interactive device or localizing an existing design for a different culture, a number of critical needs of older and disabled users should be addressed: the need for communication with and through devices, the need for mobility, and the need for personal safety. We consider two approaches for designing home appliances to address such needs for users: simulation and standardization.

Simulations can be used to help designers and developers understand the needs of disabled and older users and possibly the needs of users from other cultures. For example, the effects of having a tremor in one's hands (a common problem for older people, essential tremor is experienced by at least $4 \%$ of people over 65 [14]) may be experienced by creating a randomly jittering touch point. This allows designers to get a personal insight into older users' need for larger buttons. Visual problems can be experienced by having designers and developers were spectacles which simulate different visual conditions [15] or by distorting the screen contents [16, 17]. The latter is particularly useful for understanding how people with color vision deficiencies experience the colors in displays $[18,19,20]$. Dyslexia may be simulated by reversing, inverting and transposing characters and creating inconsistent spellings of words [21]. Limitations in dexterity may be experienced by placing small buttons on the knuckles of the hands and wearing several layers of tight rubber gloves, this impedes both mobility and sensitivity of the fingers (see Fig. 2, below). Although the simulation approach is promising it has not yet been applied to cultural design features.

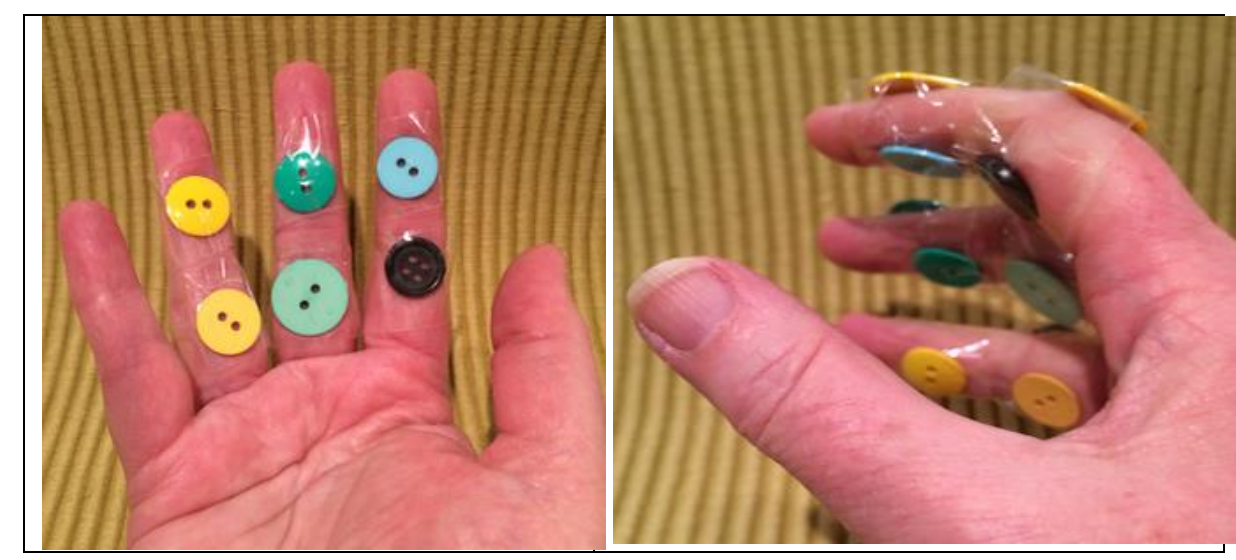




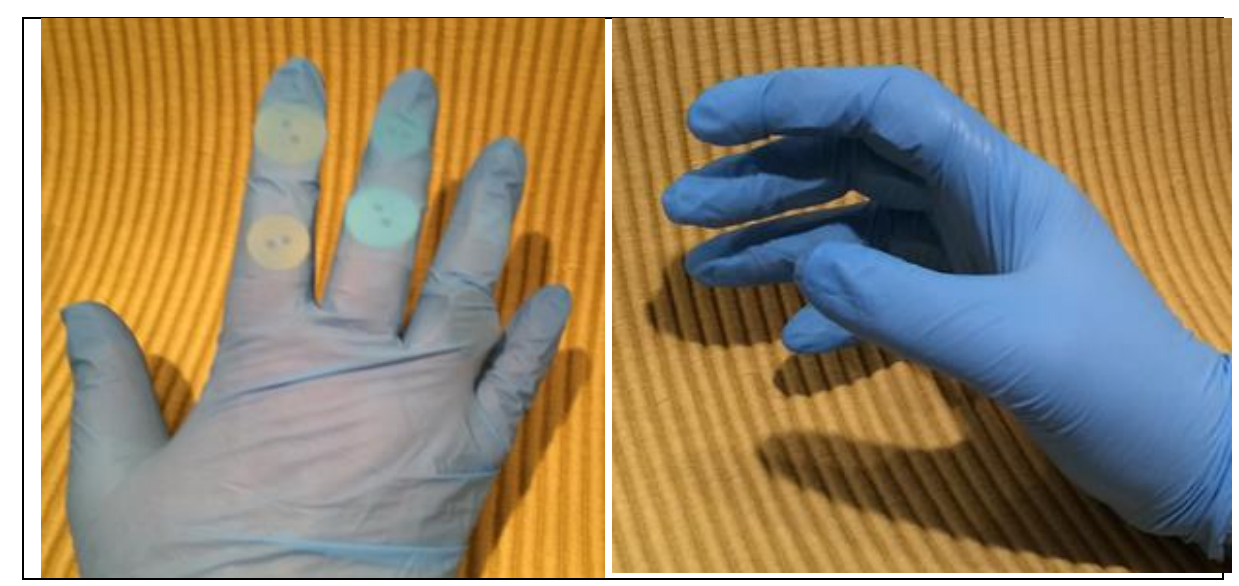

Fig. 2. Buttons and rubber gloves to simulate reduced dexterity (Photos $\mathbb{C}$ Helen Petrie and Jenny Darzentas)

Standardization of user interfaces is making good progress towards establishing the key concepts of accessibility. New work in Europe requires that those creating standards must consider whether accessibility is a concept is that is applicable to their technical committee work, and have a protocol to do this [22]. In addition, all standardization bodies revise standards periodically, meaning that theoretically, older standards will be reviewed in the light of accessibility. In particular, International Organization for Standardization (ISO) standards are subject to review and revision every five years. Thus, existing standards are updated if there are experts available to do the work. However, a problem for variations in accessibility due to cultural factors, is that even ISO, whose member countries vote for approval of standards, does not represent the cultural variations throughout the world, due to a lack of experts familiar with cultural and accessibility requirements. In addition, there normally need to be strong background economic reasons to be part of standards initiatives, that are traditionally dominated by trade and industry and less by consumer representatives. Thus, for instance in a country that does not manufacture home appliances, it is less likely to find manufacturers aware of cultural factors to do with accessibility. Similarly, consumer organizations with expert localized information about cultural factors impacting accessibility may not have an interest or the funding to participate in these standardization activities.

In the United States, the National Federation of the Blind [23] have repeatedly requested that the accessibility needs of blind users be taken into account especially in the design of home appliances and consumer electronics, and called for a Home Appliance Accessibility Act [24]. In Germany, an organization representing blind people [25] is campaigning directly to manufacturers to consider accessibility in the products they design. This is because accessibility for home appliances and consumer electronics has not yet been legally regulated, and the draft regulations about accessibility requirements for products and services in the European Accessibility Act (EAA) [26] exclude these two product groups, in spite of the recommendation from the 
expert group on standardization [22] to prioritize accessibility concerns including domestic appliances and consumer electronics.

\section{Topic 2: Cross cultural accessibility issues in India}

Another set of issues relate to language differences and the provision of assistive technologies and accessibility of digital materials. People from cultures with languages which are not widely spoken current face many barriers in access to technologies, both mainstream and assistive, due to such language issues. For example, India has 22 official languages and 12 different scripts for writing them. But in total, India has over 780 languages and over 60 different scripts [27]. The core technologies of text-tospeech synthesis, automatic speech recognition and optical character recognition software are widely used in assistive technologies to make them accessible to people with print disabilities and older people. Such assistive technologies include screen readers, screen magnifiers, and electronic braille displays. But in India these core technologies are only available for a handful of the official languages, let alone the total range of languages. Thus, assistive technologies are not available to many disabled and older Indians at all, or only in their second or third language, and not their preferred native language.

A further aspect of the language issue and accessibility is the availability of digital content in Unicode. Unicode is a computing industry standard for the consistent encoding, representation, and handling of text expressed in most of the world's writing systems. In particular, Unicode allows websites to render text in different writing systems and assistive technologies to convert text to speech and vice versa. It is very important that these digital codes are universally used. The digital code for any specific character must be the same all over the world. To achieve this the Unicode Consortium has published a standard of all possible characters of all languages of the world along with their digital codes [28]. For example, all the Indian languages are part of this Unicode standard.

The problems start with the development of different fonts. Many companies and organizations which develop fonts do not follow the scheme proposed by the Unicode Consortium. For example, the digital code in the Unicode standard for the Hindi character "ka" is 0915 whereas the Kruti Dev font (widely used in northern India, particularly in official documents) assigns the digital code 0064 to the same character. In the Unicode standard, 0064 is assigned to the Roman alphabet lower case "d". If we try to convert a Hindi document created in Kruti Dev font into braille, then the braille conversion software will convert this character into braille dots 145 (N.B. the correct set of braille dots for the Hindi character "ka" is 13, not 145). On the other hand, if you type a Roman alphabet lower case "d" into a digital device and apply the Kruti Dev font to it, then it will display the Hindi character "ka". However, screen reading software will still read it as " $\mathrm{d}$ ". One important consequence of these issues is that when an organization in India wishes to create a braille version of a document for blind readers, the electronic version of the document cannot be used, instead the document first needs to be re-typed completely. Thus, one of the great advantages of electronic documents is lost. 
The Unicode issues also affect optical character recognition (OCR) processes, one of the basic technologies used to create digital content, and one which has made a great difference to the accessibility of material for many people with print disabilities. A digital library cannot come into existence if there is no OCR software for a particular written language. The digital content gets its real power only when the characters of the content is stored as digital codes and not as an image of the printed paper. Only after digital coding of the characters do we get the flexibility of the digital content, such as representation in different media (e.g. audio, braille, even simple font changes such as large print). Creating digital content without OCR again means re-typing all the material and denies print disabled people a key component of their independence, the ability to OCR documents themselves. OCR is available for more that 100 languages across the world. Unfortunately, India languages largely lack this benefit. There is no OCR software for most Indian languages, barring a few which are very recent innovations.

As a measure of the situation in India with regard to problems with its multilingual culture, a number of articles about web accessibility have recently appeared which mention this issue. This is particularly important due to interest in India in transferring services to digital forms, most lately fuelled by the Indian Government's Digital India [29] campaign launched in 2015.

In a case study looking at the accessibility of e-portals [30], Clemmensen and Katre collected data from state government web portals in India and examined across seven parameters, including accessibility, for which they used 18 criteria. Only two portals met the accessibility requirements, as judged as meeting at least half of the 18 criteria. However, the authors noted that since 20 of the 28 portals were in the English language, this in itself probably posed considerable accessibility barriers.

In a report that examined Indian Government websites [31], amongst 7,800 websites in total, only $3 \%$ were judged as accessible and the report commented that "websites which are not developed in accordance with the Web Content Accessibility Guidelines (WCAG) 2.0 will remain inaccessible to over 50 per cent of India's population comprising persons with disabilities, elderly and illiterate persons, linguistic minorities and persons using alternate platforms like mobile phones". The report highlighted examples of accessibility errors common on the websites, one of which was the tendency to present important information written in a different language and script as an image, meaning it would be skipped over by screen readers.

A study of 48 Indian banking websites [32] was undertaken in 2014, testing the sites for accessibility using both WCAG 1.0 [33] and WCAG 2.0 [34]. Accessibility of the banking websites was low: only $25 \%$ conformed to the minimum conformance level with respect to WCAG 1.0 (Level A), and none conformed to the minimum conformance level with respect to WCAG 2.0. The authors explained that this is a problem, since although the internet banking sector in India is still new, the change from cash transfer to bank transfer of welfare payments, will mean a greater need to use internet banking. Amongst the accessibility problems found, the authors noted that that a banking website should have content in appropriate regional languages. They concluded that there is a need to develop a multilingual model for the Indian context. The imperative for the banking services to be accessible is becoming a matter of urgency as the impact of the Indian government's digital push on the economy becomes reality. Kumar Srinivasan, a leading digital entrepreneur has noted that "since the 
government's push for a cashless economy with the announcement for demonetisation in India, we have seen a rapid transformation and growth in the digital payments segment. With an unbanked population of over 250 million Indians and the country moving towards 520 million smartphone users by 2020, it is a wise move by the government to incentivise digital payments to encourage consumers to shift to "cashless" and increase bandwidths to reach out to a larger populace" [35].

Ismail et al [36] in investigating accessibility and readability features of the 20 most frequented websites in India, commented that as there were no tools and metrics for readability for languages spoken in India, they had used six different methods in their study, but these were all developed for the English language. They noted a serious gap in the availability of such tools and that there is a strong need to develop readability measures or tests that are language independent. If the language independent route is not possible, there need be efforts to build metrics which would harness the specific features of languages.

Even the official Digital India initiative is struggling with the issue of even the national languages in India as evidenced by the accessibility statement of Digital India, the following notice appears "Digital India is working towards making its portal accessible for persons with disabilities, however currently ... information provided in Hindi language is ... not accessible" [37].

Tackling the multilingual problem of India is an ongoing process: the Centre for Development of Advanced Computing (C-DAC) [38], the research and development organisation of the Ministry of Electronics and Information Technology has been working on this problem for over 25 years. The statement on their webpage about multilingual technology explains their motivations: "India is a unique country in the world having 22 scheduled languages besides heritage languages and over one hundred widely used languages with different scripts. Despite a very impressive growth of computers and the Internet over the past few decades, most of the content on the Internet and most of the ICT based solutions in India are still available only in English. This is in stark contrast to the ground reality as hardly $10 \%$ of Indians use English as a language for communication. C-DAC realized long ago that penetration of IT to masses is possible in India only if we develop tools and technologies to overcome this language barrier" [39].

\section{Topic 3: Methods of working with disabled and older users in different cultures}

A final problem to be considered is that of the methods that designers of new technologies use when working with potential users of those technologies who are from different cultures. Designers and HCI practitioners are typically taught to use a usercentred design lifecycle [40] or a "double diamond" design methodology [41]. Both these methodologies involve working closely with users with specific methods such as questionnaires, interviews, focus groups, card sort exercises and so on [42]. However, all these methods have been developed in North America and Europe and users from other cultures may interpret them differently. For example, presented with a prototype interactive system, potential users from some cultures may think it inappropriate to openly critique the design of the system, when it has been developed and presented by 
authoritative experts. Hofstede [43] would explain this by appealing to the "power distance" dimension of different cultures.

A particular case in point is the extensive use of Likert items in questionnaires about usability and user experience of interactive technologies, for both mainstream users as well as disabled and older users. On Likert items, respondents specify their level of agreement or disagreement on a symmetric agree-disagree scale for a series of statements. Thus, the range captures the intensity of their agreement or disagreement with a given item [44]. A well-known example of the use of Likert items is the System Usability Scale (SUS) [45] which asked respondents to indicate their agreement to a series of 10 statements about the interactive system they have just experienced on 5point Likert items such as "I think that I would like to use this system frequently". Although the SUS is much criticized [46], it is still widely used and fulfils a clear need in the quick and simple evaluation of interactive systems [47]. The cultural specificity of the SUS has been considered, but only for people completing it in English, for whom some of the wording may be difficult [48].

A much more fundamental problem is how people from different cultures interpret the Likert system of varying agreement/disagreement. There is a considerable history of research on this problem in cross-cultural psychology, where it is sometimes known as the "extreme response style" (ERS), a phenomenon noted as long ago as 1946 [49]. ERS is the extent to which respondents will use the extremes of a rating scale, as opposed to tending towards the middle of the scale. Variations of the phenomenon are the tendency to answer positively as opposed to negatively in relation to items. Some examples of cultural differences in ERS which have been established include: greater ERS amongst respondents in the USA compared to Korea [50]; greater ERS amongst Hispanic respondents in the USA compared to non-Hispanic respondents [51]; greater ERS amongst North American (from the USA and Canada) respondents than amongst East Asian (from Japan and Taiwan) respondents [52]; and significant differences in ERS between Australian, French, Mexican and US respondents [53].

The cultural bias in the case of Likert items is fairly easy to detect, because it is a simple quantitative measure. The cultural biases in other methods used in working with older and disabled users may be more subtle and difficult to detect, but researchers and practitioners should at least be aware of the possibility that they exist and exercise caution in extending methods to different cultures.

\section{Conclusions}

In this chapter we have begun exploring a number of issues related to cross cultural differences and cultural sensitivities have not yet received much attention in the areas of accessibility, assistive technologies, and inclusive design and methods for working with disabled and older users. This is still a very unexplored area and warrants considerable further research and discussion. However, some interesting synergies have begun to emerge. In the same way that personalization and flexibility of interaction designs can assist users with different abilities, these same principles may also be deployed to address the needs of users from different cultures and from different language groups. We welcome further exploration of these issues in a wide range of cultural and language contexts in the future. 


\section{Acknowledgements}

We would like to thank all the participants at the INTERACT 2017 Workshop "Cross Cultural Differences in Designing for Accessibility and Universal Design" for their very stimulating discussions around the topics elaborated in this chapter.

The research carried out by Jenny Darzentas for this chapter has been funded by the European Union under the Marie Skłodowska-Curie Action Experienced Researcher Fellowship Programme, as part of the Education and Engagement for inclusive Design and Development of Digital Systems and Services Project (E2D3S2, Grant No. 706396).

\section{References}

1. World Health Organization. Disability and health fact sheet. [online] Available at: http://www.who.int/mediacentre/factsheets/fs352/en/ (2015)

2. United Nations: World Population Ageing 2015 Report. [online] Available at: http://www.un.org/en/development/desa/population/publications/pdf/ageing/WPA 2015_Report.pdf (2015)

3. Mynatt, E., Melenhorst, A.-S., Fisk, A.D., Rogers, W.A.: Aware technologies for aging in place: understanding user needs and attitudes. Pervasive Computing, 3(2), 1536 - 1268 (2004)

4. Marcus, A., Aykin, N.M., Chavan, A. L., Prabhu, G.V., Kurosu, M.: SIG on one size fits all?: cultural diversity in user interface design. In Extended Abstracts on Human Factors in Computing Systems (CHI '99), 342 (1999)

5. Chavan, A.L., Gorney, D., Prabhu, B., Arora, S.: The washing machine that ate my sari - mistakes in cross-cultural design. Interactions, 16(1), 26 - 31 (2009). DOI: https://doi.org/10.1145/1456202.1456209

6. Euromonitor International. Increase in washing machine penetration. Available at: http://euromonitor.typepad.com/files/ingredients-trends-in-powder-detergents-abright-clean-future.pdf

7. http://www.worldbank.org, 17.7.2012

8. https://www.idc.com/promo/smartphone-market-share/

9. http://www.irobot.com/For-the-Home/Vacuuming/Roomba.aspx

10.http://www.gfk.com, 31.8.2012

11.Phong, K., Srou, L., Solá, J.: Mobile phone and internet use in Cambodia 2016. Phnom Penh, Cambodia: Open Institute. (2016). Available at: http://www.open.org.kh/research/phones_2016.pdf

12. Office of Communications (Ofcom). Adults' media use and attitudes. Report 2017. Available

at: https://www.ofcom.org.uk/_data/assets/pdf_file/0020/102755/adults-media-useattitudes-2017.pdf

13.Loitsch, C., Weber, G., Kaklanis, N. et al.: A knowledge-based approach to user interface adaptation from preferences and for special needs, User Modelling and User-Adapted Interaction, 27(3-5), 445-491 (2017). Available at: https://doi.org/10.1007/s11257-017-9196-Z 
14. Thanvi, B., Lo, N., Robinson, T.: Essential tremor - the most common movement disorder in older people. Age and Ageing, 35(4), 344 - 349 (2006) DOI: https://doi.org/10.1093/ageing/afj072

15.http://www.inclusivedesigntoolkit.com/csg/csg.html

16.http://www.inclusivedesigntoolkit.com/simsoftware/simsoftware.html

17.https://webaim.org/simulations/lowvision

18.http://www.color-blindness.com/

19. coblis-color-blindness-simulator/

20.http://www.vischeck.com/vischeck/

21.https://webaim.org/simulations/dyslexia

22.CEN-CENELEC Design for all Protocol, Mandate 473 Available at: https://www.cencenelec.eu/standards/Sectors/Accessibility/DesignForAll/Pages/ default.aspx

23.https://nfb.org/

24. Monitor. Home Appliance Accessibility Act. Available at: https://nfb.org/images/nfb/publications/bm/bm12/bm1203/bm120305.htm

25.Groenewold, H., Dipp, A. : IFA 2016: Accessible Household Appliances and Consumer Electronics http://www.dvfr.de/fileadmin/user_upload/DVfR/Downloads/Internationales /DBSV_Documentation-IFA-2016_English.pdf (2017)

26. European Accessibility Act http://ec.europa.eu/social/main.jsp?catId=1202

27. Bhasha Research and Publication Centre. The People's Linguistic Survey of India. Available at: http://www.peopleslinguisticsurvey.org/ (2011).

28. The Unicode ${ }^{\circledR}$ Standard. [online]. Available at: http://www.unicode.org/standard 29.https://en.wikipedia.org/wiki/Digital_India

30.Clemmensen, T., Katre, D.: Adapting e-gov usability evaluation to cultural contexts. In Buie, E. \& Murray, D. (Eds) Usability in government systems: User Experience Design for Citizens and Public Servants, Morgan Kaufman (2012)

31. Narasimhan N. Accessibility of government websites in India: a report. Available at: http://cis-india.org/accessibility/accessibility-of-govt-websites.pdf (2013)

32. Kaur, A., Dani, D.: Banking Websites in India: an accessibility evaluation. Computer Society of India Transactions on Information and Communications Technology 2(1), 23-34 (2014)

33.W3C. Web content accessibility guidelines 1.0. Available at: https://www.w3.org/TR/WAI-WEBCONTENT/

34.W3C. Web content accessibility guidelines 2.0. https://www.w3.org/TR/WCAG20/

35. Tejaswi M.: India's will to go digital evident in budget FY19, Friday, 2 February $2018 \mathrm{http} / / /$ www.mydigitalfc.com/companies-and-markets/india $\% \mathrm{E} \% 80 \% 99 \mathrm{~s}-$ will-go-digital-evident-budget-fy 19

36.Ismail, A, Kuppusamy, K.S. , Kumar, A., Ojha, A.K.: Connect the dots: Accessibility, readability and site ranking - An investigation with reference to top ranked websites of Government of India. Journal of King Saud University Computer and Information Sciences https://doi.org/10.1016/j.jksuci.2017.05.007 (2017)

37.http://www.digitalindia.gov.in/content/accessibility-statement

38.https://www.cdac.in/

39.https://cdac.in/index.aspx? $\mathrm{id}=$ mlingual 
40.https://www.usability.gov/what-and-why/user-centered-design.html

41.https://www.designcouncil.org.uk/news-opinion/design-process-what-doublediamond

42.Cairns, P. Cox, A.L.: Research Methods for Human-Computer Interaction. Cambridge University Press (2008)

43.Hofstede, G., Hofstede, G.J., Minkov, M.: Cultures and organizations: software of the mind (3rd edition). McGraw Hill (2010)

44.https://en.wikipedia.org/wiki/Rensis_Likert\#Likert_scale

45. Brooke, J.: SUS: a "quick and dirty" usability scale. In P.W. Jordan, B. Thomas, B.A. Weerdmeester, I.L. McClelland (Eds.), Usability evaluation in industry, London: Taylor and Francis (1996).

46. Sauro, J.: 10 things to know about the System Usability Scale (SUS). Available at: https://measuringu.com/10-things-sus/

47.Bangor, A., Kortum, P.T, Miller, J.T.: An empirical evaluation of the System Usability Scale. International Journal of Human-Computer Interaction, 24 (6), 574 - 594 (2008).

48.Finstad, K.: The System Usability Scale and non-native English speakers. Journal of Usability Studies, 1(4), 185 - 188 (2006).

49. Cronbach, L.J.: Response sets and test validity. Educational and Psychological Measurement, 6: 475 - 494, (1946).

50.Chun, K.-T, Campbell, J.B., Yoo, J.H.: Extreme response style in cross-cultural research: a reminder. Journal of Cross-Cultural Psychology, 5(4), 465 - 480 (1974).

51.Hui, C.H., Triandis, H.C.: Effects of culture and response format on extreme response style. Journal of Cross-Cultural Psychology, 20(3), 296 - 309 (1989).

52. Chen, C., Lee, S-Y., Stevenson, H.W.: Response style and cross-cultural comparisons of rating scales among East Asian and North American students. Psychological Science, 6(3), 170 - 175 (1995).

53. Clarke, I.: Extreme response style in cross-cultural research. International Marketing Review, 18(3), 301 - 324 (2001). 